\title{
Molecular monitoring of the diversity of human pathogenic malaria species in blood donations on Bioko Island, Equatorial Guinea
}

\author{
Tobias Schindler ${ }^{1,2^{*}} \mathbb{0}$, Tamy Robaina ${ }^{3}$, Julian Sax ${ }^{1,2}$, Jose Raso Bieri ${ }^{4}$, Maximilian Mpina ${ }^{1,2,5}$, Linda Gondwe ${ }^{1,2,5}$, \\ Ludmila Acuche ${ }^{4}$, Guillermo Garcia ${ }^{6}$, Carlos Cortes ${ }^{6}$, Carl Maas $^{7}$ and Claudia Daubenberger ${ }^{1,2^{*}}$
}

\begin{abstract}
Background: Malaria can be transmitted by blood transfusion from human to human and it is responsible for the majority of transfusion-transmitted infectious diseases worldwide. In sub-Saharan Africa, it had been estimated that almost a quarter of blood donations contain malaria parasites. Since rapid diagnostic tests and thick blood smear microscopy lack sensitivity for low density parasitaemia, particularly in asymptomatic adults, the most reliable method to assess the problem of transfusion-transmitted malaria are nucleic acid-based molecular approaches such as quantitative polymerase chain reaction. The study was undertaken to determine the prevalence of sub-microscopic malaria parasite infection among blood donors in Malabo, Equatorial Guinea.
\end{abstract}

Methods: Between July and August 2017, a total of 200 individual blood samples from blood donors at the Malabo Blood Bank were collected and screened by rapid diagnostic tests and thick blood smear microscopy. Retrospectively, the same samples were analysed for the presence of undetected, low-density malaria parasites using quantitative polymerase chain reaction.

Results: In comparison to $6.5 \%$ (13/200) by rapid diagnostic test and 2.0\% (4/200) by microscopy, the proportion of Plasmodium falciparum positive blood donations analysed by quantitative polymerase chain reaction was significantly higher (26\%, 52/200). Densities of P. falciparum positive blood donations were ranging from 0.06 to 3707.0 parasites/ $\mu \mathrm{L}$ with $79.6 \%$ below 100 parasites/ $\mu \mathrm{L}$ and therefore not detectable by non-molecular malaria diagnostic tests. qPCR based species identification revealed that P. falciparum was the dominating species responsible for $88.1 \%(52 / 59)$ of positive blood donations, followed by Plasmodium malariae $(15.3 \%, 9 / 59)$ and Plasmodium ovale $(3.4 \%, 2 / 59)$.

Conclusions: This study confirms that in malaria endemic settings, sub-patent malaria infections among blood donors are prevalent. In blood collected from healthy donors living in Malabo, P. falciparum, P. malariae and P. ovale parasites were identified. Currently widely used malaria diagnostic tools have missed more than $75 \%$ of $P$. falciparum containing blood donations, demonstrating the value of quantitative polymerase chain reaction to reliably detect low density P. falciparum infections. Since the availability of molecular diagnostic methods in malaria endemic countries is still limited, the blood recipients living in malaria endemic countries should be treated following WHO recommendations.

Keywords: Transfusion-transmitted malaria, P. falciparum, P. malariae, P. ovale, QPCR

\footnotetext{
*Correspondence: tobias.schindler@unibas.ch;

claudia.daubenberger@swisstph.ch

${ }^{1}$ Department of Medical Parasitology and Infection Biology, Swiss

Tropical and Public Health Institute, Basel, Switzerland

Full list of author information is available at the end of the article
} 


\section{Background}

Malaria is a vector-borne parasitic tropical disease found in 55 countries globally [1]. Six Plasmodium species are known to infect humans including Plasmodium falciparum, Plasmodium vivax, Plasmodium malariae, Plasmodium ovale curtisi, Plasmodium ovale wallikeri and Plasmodium knowlesi [2]. Malaria is transmitted to the human host through the bite of a female Anopheles mosquito when the sporozoite stage is inoculated during the feeding. The parasite undergoes a pre-erythrocytic liver stage reproduction that is clinically silent and lasts 1 to 2 weeks before the release of merozoites into the bloodstream. These merozoites rapidly infect red blood cells, starting the asexual blood stage whereby the parasite undergoes cycles of asexual replication that include the ring, trophozoite, and schizont stages leading to regular fever episodes [2]. In adult human populations living in malaria endemic countries, the prevalence of sub-patent malaria infections is up to $80 \%$ [3] with certain parasite clones surviving in the human host more than 300 days [4].

Diagnosis of malaria is performed in blood samples collected from potentially infected humans. The gold standard for malaria diagnosis remains light microscopy of stained blood smears. Thick blood smears provide sensitivity and thin smears allow the identification of different malaria species and the quantitation of infection [5]. Rapid diagnostic tests (RDTs) are widely used since they offer less dependence on the availability of laboratory infrastructure, and can also be employed by inexperienced health workers [6]. RDTs are often based on parasite-derived histidine-rich protein 2 (PfHRP2) for sensitive and specific detection of $P$. falciparum, and lactate dehydrogenase enzyme (LDH) or aldolase for a PanPlasmodium detection of all human infective malaria species [7]. The lower limit of detection (LOD) for microscopy is between 50 and 500 parasites/ $\mu \mathrm{L}$ depending on the expertise of the microscopist [5] and 100 parasite $/ \mu \mathrm{L}$ for the PfHRP2 based P. falciparum RDTs [8]. Differential diagnosis of $P$. ovale, $P$. malariae, $P$. vivax and $P$. knowlesi is hampered by the low density blood stage infections and the difficulty in distinguishing these species based on morphology of trophozoites using microscopy [9].

Nucleic acid amplification technologies (NATs) are more expensive and require advanced laboratory infrastructure but provide much better sensitivity. The parasites' multi-copy $18 \mathrm{~S}$ small subunit ribosomal deoxyribonucleic acid (rDNA) and/or its highly expressed ribonucleic acid (rRNA) is the most frequently used biomarker for NATs [10]. Different methods for 18S rDNA/ rRNA detection are currently in use with a great variation in sensitivity. Qualitative methods targeting $18 \mathrm{~S}$
rDNA, such as nested polymerase chain reaction (PCR) [11] and LAMP [12], reach LODs of 0.1-10 parasites $/ \mu \mathrm{L}$. The use of quantitative PCR (qPCR) [13], does not only allow absolute quantification of infections but also lowers the LOD to less than 1 parasite $/ \mu \mathrm{L}$. The use of Reverse Transcription quantitative PCR (RT-qPCR), amplifying total nucleic acids (RNA and DNA) of the 18S genes [14$16]$, and qPCR assays targeting alternative multi-copy genomic sequences [17] further increase sensitivities of NATs. Apart from increased sensitivity of NATs, molecular detection using PCR has improved species discrimination compared to either microscopy or RDTs [18].

Screening for transfusion transmitted infections should follow the World Health Organization (WHO) recommendations which include searching for chronic infectious diseases including HIV-1, HIV-2, Hepatitis B, Hepatitis C and Treponema pallidum (syphillis) [19]. Based on the local epidemiological infectious disease situation, the WHO recommends that blood donations should also be screened for malaria [19, 20]. Malaria can be efficiently transmitted by blood transfusion from human to human and is undoubtedly responsible for the majority of transfusion transmitted diseases in the world [21]. The rate of transfusion-transmitted malaria (TTM) in malaria endemic sub-Saharan regions is estimated between 14 and 28\% [22]. Several reports describe that $P$. falciparum [22], P. vivax [23], P. malariae [24], P. knowlesi [25] and $P$. ovale [26] can be transmitted either through blood donations or solid organ transplantations.

Malaria species circulating in Equatorial Guinea include $P$. falciparum, $P$. malariae, $P$. ovale and $P$. vivax [27-29]. The Bioko Island Malaria Control Project (BIMCP) started in 2004 with the aim to reduce malaria transmission and to control the burden of disease and has received substantial funding from the Government of Equatorial Guinea and private donors including Marathon EG Production Limited, Noble Energy, and Atlantic Methanol Production Company. The malaria control strategy of the BIMCP rests on vigorous vector control measures, effective case management, prevention of malaria during pregnancy, behavioural change communications and regular monitoring and evaluation [30]. The BIMCP has had significant success in reducing $P$. falciparum parasite prevalence in the 2-14 years old children and adolescent from 45\% (95\% CI 40-50\%) in 2004 (baseline) to $12.1 \%$ (95\% CI 11.2-13.3\%) in 2016 based on annual Malaria Indicator Survey (MIS) data [30].

Since RDTs and microscopy lack sensitivity for lowlevel parasitaemia, particularly in asymptomatic adults, the most reliable method to assess the problem of TTM are NATs such as qPCR. This study has set out to analyse a selection of 200 blood samples from the blood bank in Malabo using qPCR to identify sub-patent $P$. falciparum 
infections that cannot be detected by commonly used RDTs and microscopy. In addition, the presence of other TTM causing Plasmodium species was analysed using a newly developed single tube multiplex qPCR assay.

\section{Methods}

\section{Study site and participant recruitment}

The study was conducted at the Central Blood Bank in Malabo on Bioko Island. Bioko Island, located in Westcentral Africa and home of Equatorial Guinea's capital city Malabo, is $32 \mathrm{~km}$ from the coast of Cameroon. The approximately 250,000 people, who mainly reside in Malabo are at risk of malaria year-round [30]. Adults willing to donate blood and who are Hepatitis B, Hepatitis $\mathrm{C}$, and HIV negative were eligible for blood donation. Microscopy and RDT (CareStart ${ }^{\mathrm{TM}}$ Malaria HRP2/ pLDH Pf/PAN Combo) were used for malaria screening at the blood bank and the results were kept at the blood bank until the end of qPCR analysis. The Malabo Central Blood Bank processes around 100 donors per month; the majority being donors assisting friends and families during emergency situations, and the remainder are donors that voluntarily donate blood on a regular basis. The blood bank is run by a public-private cooperation between the Ministry of Health and Social Welfare, the University of Valencia Hospital, and funded by the AGEM-GUINEA company.

\section{Sample collection for molecular diagnostics}

Between July and August 2017, a total of 200 individual blood donations from routine visitors were selected to conduct this study. $1 \mathrm{~mL}$ of whole blood was collected in EDTA tubes as part of the usual blood donation procedures in the blood bank. The blood was immediately used for microscopy and RDT, with the remaining blood immediately stored at $-20{ }^{\circ} \mathrm{C}$. The samples were transported in a cooling box to the laboratory of the Equatorial Guinea Malaria Vaccine Initiative (EGMVI) in Malabo. The laboratory infrastructure of the EGMVI, located on the premises of the La Paz Hospital in Malabo, which conducted the first clinical trial in the history of the country [31], was used for qPCR analysis of the blood donations.

The 200 blood samples, from healthy, Hepatitis B, Hepatitis C, and HIV-1 and HIV-2 negative blood donors were analysed for the presence of possibly undetected, low-level malaria parasites using high-sensitive qPCR assays. DNA extraction was done manually from $180 \mu \mathrm{L}$ whole blood using Quick-DNA Miniprep kits (Zymo Research, Irvine, USA) following manufactures' guidelines and DNA was eluted with $50 \mu \mathrm{L}$ Elution Buffer. DNA samples were kept at $-20{ }^{\circ} \mathrm{C}$ prior to qPCR analysis.
qPCR for Plasmodium screening and quantification (PlasQ assay)

Plasmodium spp. and P. falciparum parasites were quantified using the PlasQ assay. This assay consists of two independent Plasmodium targets combined in a multiplex assay. The Pan-Plasmodium 18S rDNA sequence (Pspp18S) [13, 32], and the P. falciparum-specific acidic terminal sequence of the var genes (PfvarATS) [17] were used for detection and quantification of parasites. The human RNaseP (HsRNaseP) gene served as an internal control to assess the quality of DNA extraction and qPCR amplification.

Amplification and $\mathrm{qPCR}$ measurements were performed using the Bio-Rad CFX96 Real-Time PCR System (Bio-Rad Laboratories, California, USA). The thermal profile used for PlasQ qPCR is as follows: $60 \mathrm{~s}$ at $95^{\circ} \mathrm{C}$; 45 cycles of $15 \mathrm{~s}$ at $95^{\circ} \mathrm{C}$ and $45 \mathrm{~s}$ at $57^{\circ} \mathrm{C}$. Each reaction contained $2 \mu \mathrm{L}$ DNA and $8 \mu \mathrm{L}$ reaction master mix containing $1 \times$ Luna Universal Probe qPCR Master Mix (New England Biolabs, Ipswich, USA) and $1 \times$ PlasQ Primer Mix (Additional file 1). All qPCR assays were run in duplicates with appropriate controls including NonTemplate Control [NTC] and P. falciparum 3D7 DNA as positive control [PC]. The mean Cq of the two replicates was reported and in case of one qPCR replicate interpreted as malaria positive and the other replicate negative, then the assay had to be repeated to arrive at an unequivocal result.

Using the first WHO International Standard for $P$. falciparum DNA Nucleic Acid Amplification Techniques (NIBSC code: 04/176), a serial dilution ranging from 0.05 to 10,000 parasites $/ \mu \mathrm{L}$ was prepared and used for quantification of $P$. falciparum. The WHO standards were run as duplicates in two out of the total 13 qPCR runs conducted.

\section{qPCR for Plasmodium species identification (PlasID assay)}

Several published [17, 33-37] and unpublished qPCR assays, detecting $P$. falciparum, $P$. malariae, $P$. ovale, $P$. vivax and $P$. knowlesi were evaluated and the best performing primer and probes for each Plasmodium species were combined into a new multiplex assay (PlasID). The primers and probe combinations used for this novel pentaplex malaria qPCR assay is provided in Additional file 1.

Amplification and qPCR measurements were performed using the Bio-Rad CFX96 Real-Time PCR System (Bio-Rad Laboratories, California, USA). The thermal profile used for the PlasID qPCR is as follows: $15 \mathrm{~min}$ at $95{ }^{\circ} \mathrm{C}$; 45 cycles of $15 \mathrm{~s}$ at $95{ }^{\circ} \mathrm{C}$ and $60 \mathrm{~s}$ at $55^{\circ} \mathrm{C}$. Each reaction contained $2 \mu \mathrm{L}$ DNA and $8 \mu \mathrm{L}$ reaction master mix containing $1 \times$ HOT FIREPol ${ }^{\circledR}$ Probe qPCR Mix Plus 
(Solis Bio Dyne, Tartu, Estonia) and $1 \times$ PlasID Primer Mix (Additional file 1). All qPCR assays were run in duplicates with appropriate controls including Non-Template Control [NTC] and DNA of six Plasmodium species as positive controls [PC]. DNA controls for P. falciparum, $P$. knowlesi, P. malariae, P. vivax, $P$. ovale curtisi and $P$. ovale wallikeri were used to evaluate species specificity of the PlasID assay. The positive controls were reconfirmed by two commercial qPCR assay (GeneFinder ${ }^{\mathrm{TM}}$ Malaria RealAmp $P f / P v / P k$ and $P f / P m / P o$ ). Samples with a $\mathrm{Cq}$ value less than 45 for any of the five Plasmodium targets were considered positive for the corresponding Plasmodium species.

\section{Data management and analysis}

Geneious version 8.1.9 (Biomatters Ltd, Auckland, New Zealand) was used for sequence alignments and oligonucleotide designs. $\mathrm{Cq}$ values were obtained from the Bio-Rad CFX96 Manager 3.1 software (Bio-Rad Laboratories, California, USA) and transferred to a Microsoft Access based in-house database designed for storage and analysis of qPCR data. Cq values were transformed to parasite densities and linked to patient's metadata collected at the blood bank. Categorical variables were compared by Fisher's exact test and continuous variables by Mann-Whitney using GraphPad Prism version 7.00 for
Windows (GraphPad Software, La Jolla, USA). P values $<0.05$ were considered to be significant.

\section{Results}

Development and implementation of multiplex qPCR assays for malaria screening

Two independent qPCR assays were systematically used for the screening and identification of malaria parasites. The use of two consecutive qPCR assays maximizes the amount of information generated by the analysis. The first assay (PlasQ), applied to all samples, was designed to identify and quantify $P$. falciparum and/or non-falciparum species. Additionally, the internal control serves as a general control for quality and performance of the DNA extraction and $\mathrm{qPCR}$ reaction. The performance of the PlasQ assay is shown in Fig. 1a. For both targets, PfvarATS and Pspp18S, the Cq values for different parasite densities, ranging from 0.05 to 10,000 parasites/ $\mu \mathrm{L}$ were obtained (Pearson $\mathrm{r}-0.9969$ and -0.9968 , respectively). Similar qPCR efficiencies for both targets were observed (87.6\% and $89.7 \%$ ). The PfvarATS target did detect two additional samples carrying low parasite densities $(0.1$ and 0.05 parasites $/ \mu \mathrm{L})$ resulting in 10 times lower LOD compared to the Pspp18S target. The second assay (PlasID), which is applied to all Plasmodium spp. positive samples, was designed for rapid identification
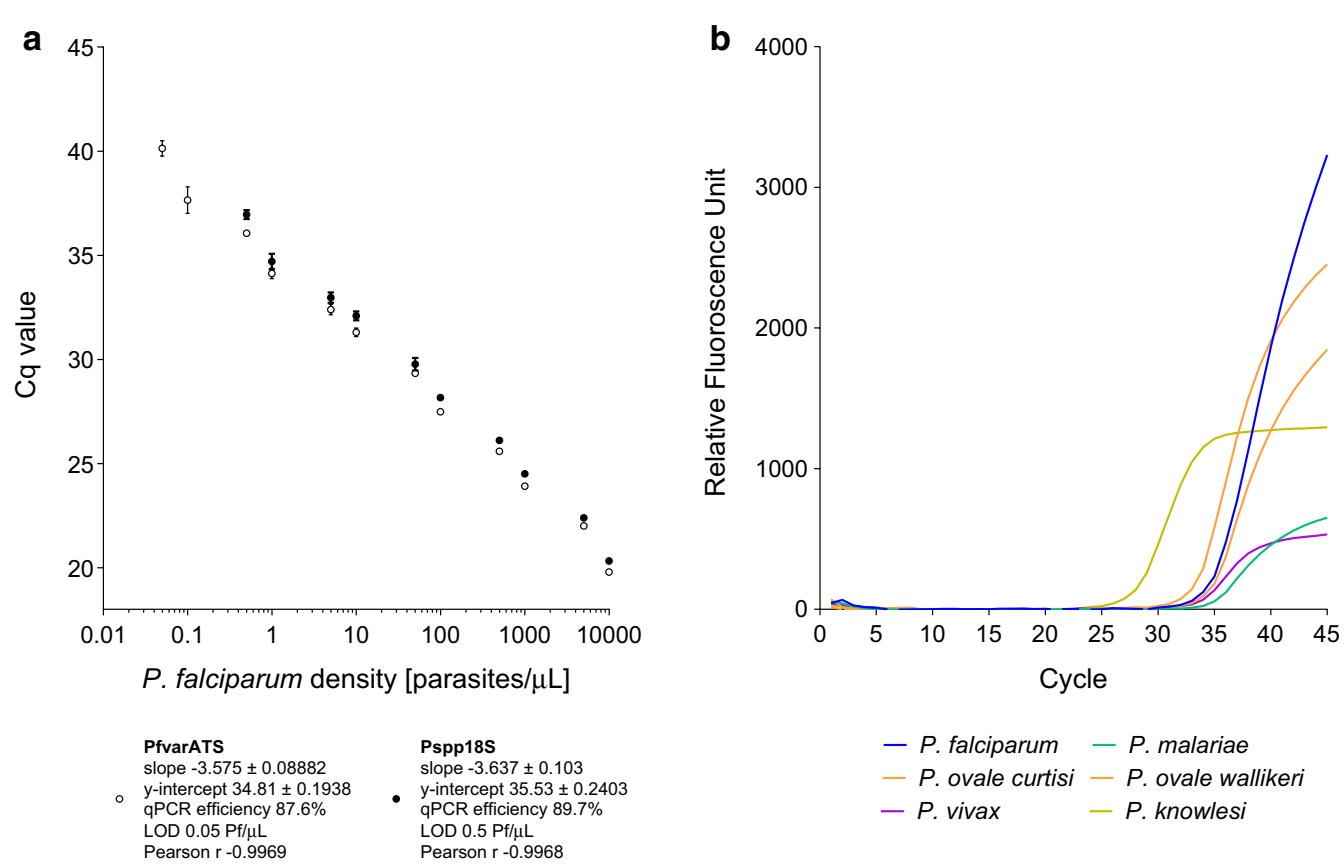

Fig. 1 Analytical performance of PlasQ (a) and PlasID (b) assay. a Correlation of P. falciparum standards and the Cq values for both targets, Pspp18S (black circles) and PfvarATS (white circles) of the PlasQ assay. Based on these quadruple replicates of the WHO standards, LODs and qPCR efficiencies were calculated. $\mathbf{b}$ The ability of the PlasID assay identifying five different malaria species is shown by a representative, composite amplification plot. DNA from the six Plasmodium species were analysed in separate tubes during the same qPCR experiment 
of five different malaria causing Plasmodium species. Performance of the PlasID assay was assessed using well-defined clinical samples as references and this novel assay's ability of identifying $P$. falciparum, $P$. malariae, $P$. ovale curtisi, $P$. ovale wallikeri, $P$. vivax and $P$. knowlesi is demonstrated in Fig. 1b.

\section{Baseline characteristics of blood donors attended Malabo Central Blood Bank}

Blood donor characteristics are given in Table 1. All donors were Equato-Guineans living in Malabo on Bioko Island except two persons, one originating from neighbouring Douala, Cameroon and the other from the United States. The majority were male $(88.5 \%)$ with a median age of 32 years, ranging from 18 to 57 years. Seventy-seven donations were from voluntary blood donors and 123 donations were from replacement donors, who donate blood when required by a member of the patient's family or community (Table 1). Except for the blood pressure, no significant difference in relation to gender, age, weight, pulse and anaemia status between these two donor groups were observed.

\section{Prevalence of malaria positive blood donations}

The flow diagram depicting the sequential malaria diagnostic tools applied to the blood donations is summarized in Fig. 2. In the Malabo blood bank, all samples were screened using thick blood smear microscopy and RDT. Microscopy identified four $P$. falciparum positive donations, while RDTs detected 13 malaria positive donations. Three donations were positive for both antigens, the P. falciparum specific HRP2 and the Plasmodium spp. LDH antigens. The remaining ten donations were positive for HRP2 only. Microscopy and RDT positive blood donations were not considered for donation

Table 1 Comparison of health characteristics between voluntary and replacement donors

\begin{tabular}{|c|c|c|c|}
\hline & $\begin{array}{l}\text { Voluntary } \\
\text { donors }\end{array}$ & $\begin{array}{l}\text { Replacement } \\
\text { donors }\end{array}$ & P values $^{\mathrm{a}}$ \\
\hline Number of donors & 77 & 123 & - \\
\hline$\%$ male & $84.4 \%(65 / 77)$ & $91.1 \%(112 / 123)$ & 0.175 \\
\hline Age in years ${ }^{b}$ & $32[19-55]$ & $32[18-57]$ & 0.888 \\
\hline Weight in $\mathrm{kg}^{\mathrm{b}}$ & $73.9[51-116]$ & 72.0 [52-116.6] & 0.593 \\
\hline $\begin{array}{l}\text { Blood pressure in } \\
\mathrm{mmHg}^{\mathrm{b}}\end{array}$ & 126.7 [97.7-169.0] & 130.8 [103.6-198.9] & 0.035 \\
\hline Pulse in bpm ${ }^{b}$ & 74 [48-101] & 73 [51-102] & 0.788 \\
\hline $\begin{array}{l}\text { Haemoglobin in } \\
\mathrm{g} / \mathrm{dL}^{\mathrm{b}}\end{array}$ & 14.6 [12.2-19.6] & $14.7[6.4-18.0]$ & 0.808 \\
\hline
\end{tabular}

a Variables were compared by Fisher's exact test for categorical variables or Mann-Whitney test for continuous variables

b Values expressed as medians with ranges and were included blinded into this study sample collection on purpose to test the performance of the PlasQ assay.

Upon arrival at the EGMVI laboratory all samples were screened with the first multiplex qPCR assay (PlasQ), targeting P. falciparum (PfvarATS), Plasmodium spp. (Pspp18S) and the human RNAseP gene (as an internal control). All 200 samples amplified the internal control with $\mathrm{Cq}<28$ and were therefore eligible for further analysis. Over $70 \%(n=141)$ of blood donations were negative for any malaria species. Forty-six donations were positive for both Plasmodium targets, while six and seven donations were positive only for $P$. falciparum or Plasmodium spp., respectively. All samples $(n=53)$ with a positive amplification of the Pspp18S target were analysed with the species identification assay (PlasID). Apart from confirming $P$. falciparum $(\mathrm{n}=33)$ cases, we also found cases of $P$. malariae $(\mathrm{n}=9)$ and $P$. ovale $(\mathrm{n}=2)$. Amongst the 200 blood samples analysed, no P. vivax or P. knowlesi was detected.

The assay identified $P$. malariae or $P$. ovale in all seven samples which were positive for Plasmodium spp. and negative for $P$. falciparum, highlighting the assays ability to identify non-falciparum species. Compared to the PlasQ assay, a reduced sensitivity $(71.7 \%, 33 / 46)$ for $P$. falciparum detection using the PlasID assay was observed. The 13 P. falciparum positive samples missed by the PlasID assay had moderate parasitaemia (median of 36.9 with IQR of 1.3-380.0 parasites/ $\mu \mathrm{L}$ ) and the five samples with the highest parasitaemia were positive by RDT. None of these samples were positive for any other non-P. falciparum species.

An additional 29 malaria negative blood samples were also run with the PlasID assay to test specificity of the assay. No false positive Plasmodium spp. cases were detected, resulting in $100 \%$ specificity.

Notably, none of the non-falciparum species had been identified by microscopy or RDT. Pan-positive RDTs, which detected the Plasmodium spp. LDH, were rather associated with higher $P$. falciparum density than with the detection of non-falciparum malaria parasite species. The three HRP2 and LDH positive RDTs had a geometric mean of 916.3 parasites/ $\mu \mathrm{L}$ (range 244.1-3707.0) while the nine HRP2 only positive RDTs had a geometric mean of 106.0 parasites/ $\mu \mathrm{L}$ (range 11.2-543.3) (Table 2).

In summary, 59 blood donations were positive for at least one malaria species (29.5\%). Plasmodium falciparum was the dominating species responsible for $88.1 \%$ of positive blood donations, followed by $P$. malariae (15.3\%) and P. ovale (3.4\%). Mixed species infections were found in $6.8 \%(4 / 59)$ of the malaria positive blood donations. One blood donation carried a $P$. malariae and $P$. ovale co-infection and three donations contained 


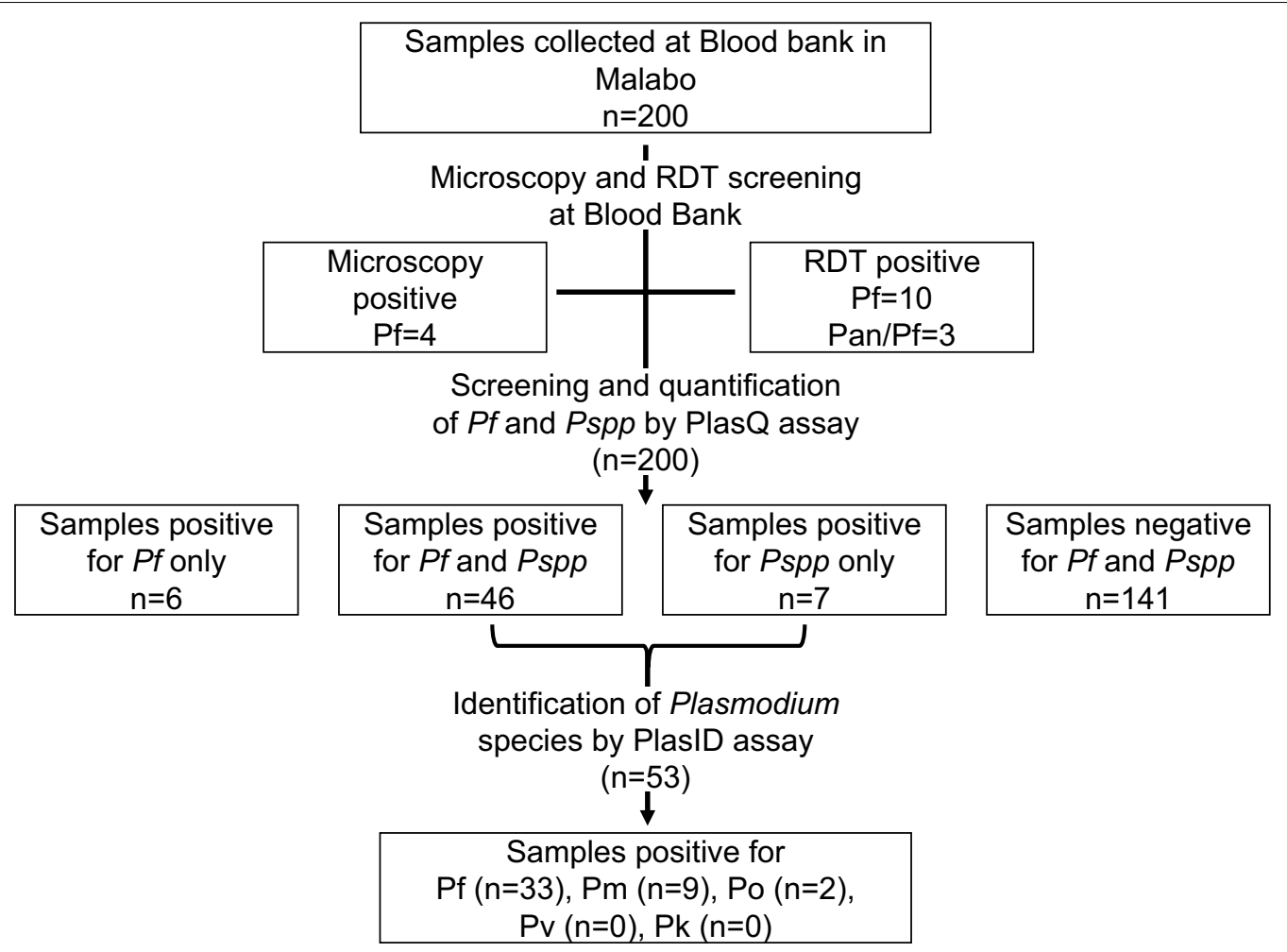

Fig. 2 Overview of blood sample analysis. Blood donations were systematically screened for the presence of malaria parasites by microscopy, RDT and qPCR assays

Table 2 Parasitaemia of blood donations detected with different diagnostic methods

\begin{tabular}{|c|c|c|c|}
\hline Diagnostic tool & Median/IQR (parasites/ $\mu \mathrm{L})^{a}$ & 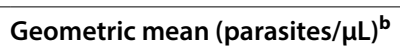 & Range (parasites $/ \mu \mathrm{L}$ ) \\
\hline qPCR-PfvarATS & $4.6[0.8-49.0]$ & $5.6[2.6-12.1]$ & $0.06-3707.0$ \\
\hline qPCR-Pspp18S & $7.8[1.3-65.1]$ & $9.5[4.5-19.8]$ & $0.07-3707.0$ \\
\hline RDT combined ${ }^{c}$ & $134.6[65.1-536.5]$ & 181.7 [68.98-478.7] & $11.16-3707.0$ \\
\hline HRP2-RDT ${ }^{d}$ & $112.5[52.2-331.2]$ & $106.0[42.4-265.1]$ & $11.16-3707.0$ \\
\hline HRP2/LDH-RDT ${ }^{e}$ & $850.4[244.1-3707.0]$ & 916.3 [31.11-26990.0] & $244.1-3707.0$ \\
\hline Microscopy & $380.1[145.4-2909.0]$ & $478.7[44.03-5205.0]$ & $112.5-3707.0$ \\
\hline
\end{tabular}

a Quartiles (Median, 25th and 75th percentile)

b Geometric mean and 95\% confidence interval

c Pf- and Pf/Pan-RDT results combined

d Pf-RDT results only

e Pf/Pan-RDT results only

a combination of $P$. falciparum and $P$. malariae. The observed co-infection proportion between $P$. falciparum and $P$. malariae was slightly higher than expected $(1.5 \%$ versus $1.2 \%, \mathrm{P}=0.037)$.

\section{Plasmodium falciparum densities in malaria positive blood donations}

Data obtained from the PlasQ assay was used to quantify $P$. falciparum positive blood donations. Parasite densities calculated based on both targets show a high correlation (Spearman r 0.894, Fig. 3). Identified non-falciparum species are indicated with green dots. Quantification based on the Pspp18S assay revealed, that the parasitaemia of these non-falciparum species is around or below 10 parasites $/ \mu \mathrm{L}$ which was supported by the high $\mathrm{Cq}$ values obtained from the PlasID assay. The geometric mean $\mathrm{Cq}$ value of the nine $P$. malariae positive samples was 36.9 (range 35.3-39.0) and the two positive $P$. ovale samples had Cq values of 37.9 and 39.8 .

A strong association between the diagnostic tests and the parasitaemia in P. falciparum positive blood 


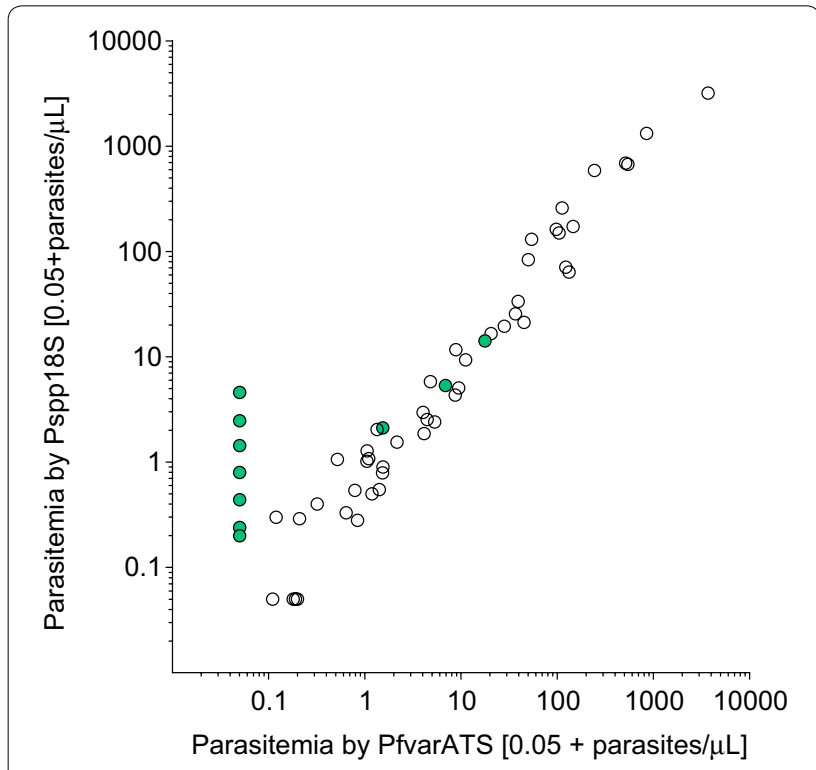

Fig. 3 Parasite densities of positive blood donations obtained from qPCR assays. Strong correlation between the two targets is observed (Spearman $r$ 0.894). The green dots highlight samples containing non-falciparum malaria species. An offset of 0.05 parasites $/ \mu \mathrm{L}$ was used donations was observed. Non-cumulative parasite density data grouped by diagnostic test is shown in Fig. 4 (scatter plot, left y-axis).

In Table 2, the parasite densities grouped by diagnostic tool are summarized. Densities of $P$. falciparum positive blood donations ranged from 0.06 to 3707.0 parasites $/ \mu \mathrm{L}$ with a median of 4.6 parasites/ $\mu \mathrm{L}$ (IQR 0.8-49.0), which is lower than the LOD for conventional diagnostic tests. Only samples with the highest parasitaemia were detectable by microscopy (381.1 [145.4-2909.0] parasites/ $\mu \mathrm{L})$ and RDTs (134.6 [65.1-536.5] parasites/ $\mu \mathrm{L})$, while the two qPCR assays detected additional infections with lower parasite densities. The median parasitaemia was 7.8 parasites/ $\mu \mathrm{L}$ (IQR 1.3-65.1) for the Pspp18S target and 4.6 parasites/ $\mu \mathrm{L}$ (IQR 0.8-49.0) for the PfvarATS target (Table 2).

\section{Performance of diagnostic methods for P. falciparum detection in blood donations}

In total, 48 previously undetected $P$. falciparum infections were identified by conducting the varATS based qPCR analysis compared to microscopy, increasing the proportion of infected blood donations from 2.0 to

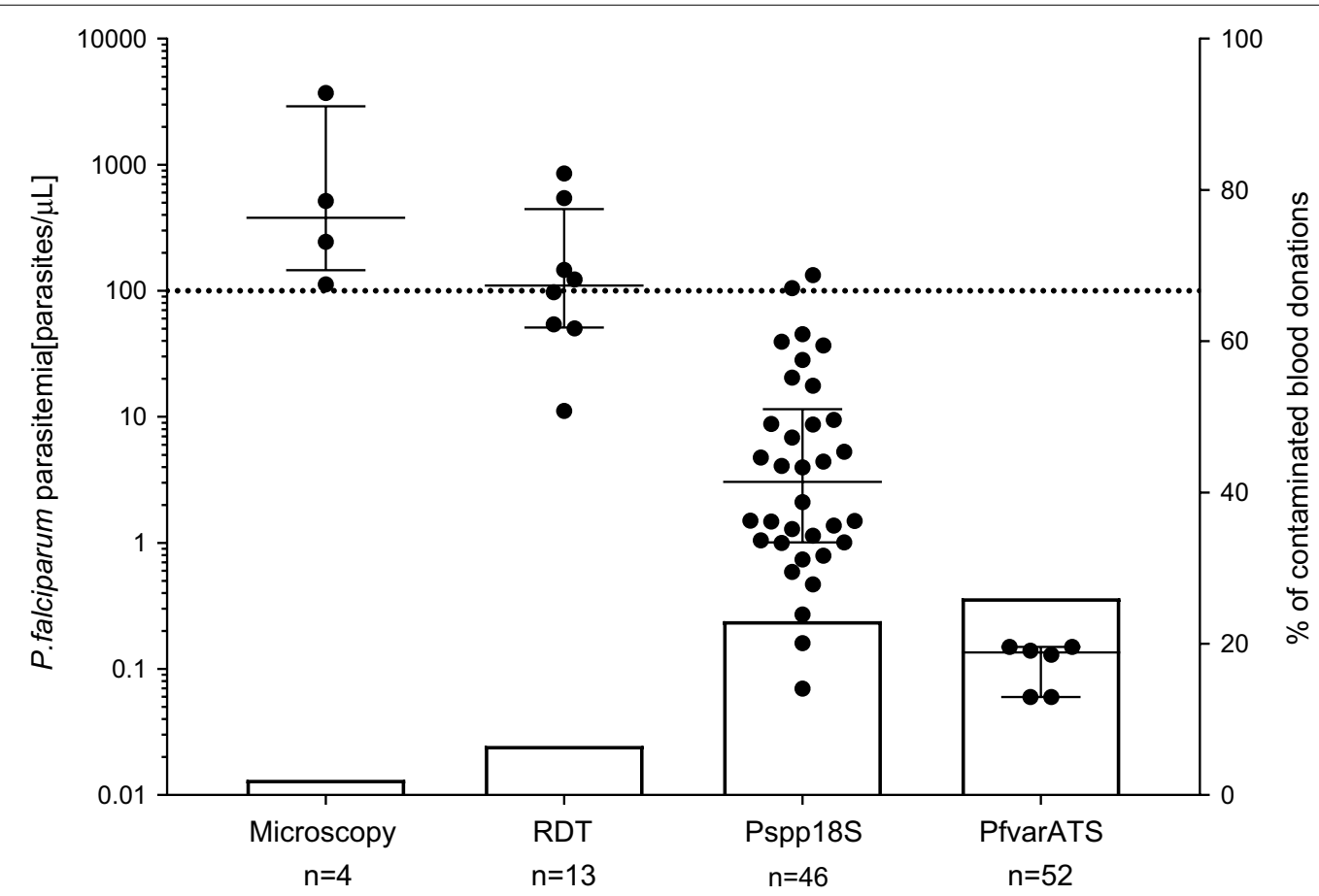

Fig. 4 Association between parasitaemia and sensitivity of diagnostic methods applied in this study. Median and interquartile ranges of non-cumulative P. falciparum parasitaemia are grouped according to different diagnostic tests used in this study (left $y$-axis, scatter plot). The dashed line at 100 parasites/ $\mu \mathrm{L}$ represents the widely accepted LOD for RDTs. Proportional rates of positive blood donations are represented as bars (right y-axis) 
$26.0 \%$. The detection rate for RDTs and $18 \mathrm{~S}$ based qPCR were $6.5 \%$ and $23.0 \%$, respectively (Fig. 4 , bar plot, right $y$-axis). This increased detection rate of the qPCR assays is also reflected in analytical sensitivities of the diagnostic tests. Using the PfvarATS qPCR results as the gold standard for P. falciparum detection, Pspp18S qPCR (88.5\%), RDT (23.1\%) and microscopy (7.7\%) have all reduced sensitivities. Specificities were for all tests above 95\%. One RDT positive sample could not be confirmed by qPCR, while the specificity for Pspp18S was reduced because of non-falciparum malaria parasite species, which are also detected (Table 3).

\section{Identification of risk factors for malaria positivity}

Malaria positive and negative donors were stratified according to the differences in the questionnaire that all blood donors must fill before proceeding to blood donation (Additional file 2). The major risk factor for being a malaria positive blood donor was reporting fever or malaria during the past 3 weeks (Additional file 2). When comparing the anthropometric measurements between malaria positive and negative donors, marginal differences in age and weight become apparent (Table 4). An increased rate of positive blood donations in replacement donors (29.3\%) compared to voluntary donors (20.8\%) was observed, as well as an increased parasitaemia among replacement donors (8.9 versus 2.1 parasites/ $\mu \mathrm{L})$. However, these differences were not statistically significant.

\section{Discussion}

This study, conducted in Equatorial Guinea, extends previous observations of presence of TTM in sub-Saharan Africa, and demonstrates the value of NAT approaches to reliably detect sub-patent $P$. falciparum and non-falciparum malaria parasites including $P$. malariae and $P$. ovale.

Table 3 Analytical comparison of methods deployed for $P$. falciparum detection

\begin{tabular}{lllll}
\hline Diagnostic tool & Sens $^{\mathbf{a}}$ & Spec $^{\mathbf{b}}$ & PPV $^{\mathbf{c}}$ & NPV $^{\mathbf{d}}$ \\
\hline qPCR-PfvarATS & \multicolumn{2}{l}{ Gold standard } & & \\
qPCR-Pspp18S & $88.5 \%$ & $95.3 \%$ & $86.8 \%$ & $95.9 \%$ \\
& {$[76.6-95.7]$} & {$[90.5-98.1]^{e}$} & {$[76.0-93.2]^{\mathrm{e}}$} & {$[91.7-98.0]$} \\
RDT & $23.1 \%$ & $99.3 \%$ & $92.3 \%$ & $79.0 \%$ \\
& {$[12.5-36.8]$} & {$[96.3-99.9]$} & {$[61.5-100.0]$} & {$[76.0-81.0]$} \\
Microscopy & $7.7 \%$ & $100.0 \%$ & $100 \%$ & $75.5 \%$ \\
& {$[2.1-18.5]$} & {$[97.54-100]$} & - & {$[74.0-76.9]$} \\
\hline
\end{tabular}

\footnotetext{
a Sensitivity and $95 \%$ confidence interval

b Specificity and $95 \%$ confidence interval

c Positive predictive value and $95 \%$ confidence interval

d Negative predictive value and $95 \%$ confidence interval

e Specificity and PPV of Pspp $18 \mathrm{~S}$ qPCR assay is reduced because non-falciparum Plasmodium species are also detected
}

Table 4 Anthropometric differences in $P$. falciparum positive versus $P$. falciparum negative blood donors

\begin{tabular}{llll}
\hline & Negative donors & Positive donors & P values $^{\mathbf{a}}$ \\
\hline Number of donors & 148 & 52 & - \\
\% male & $86.5 \%(128 / 148)$ & $94.2 \%(49 / 52)$ & 0.205 \\
Age in years & $33[18-57]$ & $29[20-55]$ & 0.013 \\
Weight in kg & $74.6[51-116.6]$ & $69.2[53-110]$ & 0.025 \\
$\begin{array}{l}\text { Blood pressure in } \\
\text { mmHg }\end{array}$ & $128.7[97.7-198.9]$ & $128.3[100.6-156.9]$ & 0.326 \\
$\begin{array}{l}\text { Pulse in bpm } \\
\text { Haemoglobin in }\end{array}$ & $74[48-102]$ & $72[51-96]$ & 0.519 \\
g/dL & $14.6[6.4-19.6]$ & $14.7[11.6-16.5]$ & 0.319 \\
\hline
\end{tabular}

a Fisher's exact test for categorical variables or Mann-Whitney test for continuous variables was applied

b Values expressed as medians with ranges

Apart from $P$. falciparum, other malaria causing species such as $P$. vivax, $P$. malariae, and $P$. ovale have been described to circulate on Bioko Island-similar to neighbouring West African countries [27, 28]. Transfusion of any of these malaria species during blood donations have the potential to cause severe disease in recipients. In subSaharan Africa, those most at risk are anaemic infants and children, pregnant women, or women who suffer from high blood losses while giving birth [25, 38, 39]. Although, there is a high frequency of TTM in malaria endemic regions, the semi-immunity of recipients may largely control parasite replication and therefore clinical outcome [40].

In malaria non-endemic countries, TTM needs also be taken into account to ensure safe blood donations [41]. Here, blood the recipients are mainly malaria naïve and the immunological control of potentially transfused parasites is non-existent resulting in in-creased risk of severe malaria disease. P. falciparum parasites have been found to survive in blood donations for up to 14 days limiting the potential to neutralize malaria during blood donation processing and storage [42].

This study demonstrates the challenges blood banks in malaria endemic countries encounter. The blood donors at the Malabo blood bank were mainly male adults within an age range of 18-57 years. Because of their pre-existing immunity, blood donors of that age tend to have low levels of parasites without exhibiting clinical symptoms. Ninety-seven percent (97.0\%) reported to be in good health at the time of blood donations. Apart from selfreported malaria and/or fever episodes within the last 3 weeks, no other risk factor had been identified based on the provided questionnaire. Since only about 3\% (6/198) had reported to have malaria and/or fever episodes, the use of this risk factor to pre-select blood donors would not be very efficient. Deferral of about a quarter of blood 
donors that are sub-patent malaria carriers in a region where blood supply shortage is eminent could be even more damaging to public health.

Conventional diagnostic tests are important tools, since they are affordable and can be applied immediately at blood banks, but lack in sensitivity limits their usefulness. Microscopy is still an important tool for malaria detection since it allows for species identification and quantification. The detection limit of an expert microscopy is close to $20-50$ parasites $/ \mu \mathrm{L}$, but for nonexperienced microscopists, it is up to tenfold higher (i.e. 500 parasites $/ \mu \mathrm{L}$ ) [5]. Currently available RDTs are based on detection of circulating, parasite derived HRP2, aldolase or lactate dehydrogenase in serum or whole blood samples. These RDTs have the ability to detect Plasmodium spp. infections with a LOD between 100 and 1000 parasites/ $\mu \mathrm{L}$ which is significantly higher when compared to NATs [43]. In addition, there is currently no single RDT available that could distinguish all five malaria species infections posing a clear limitation to comprehensive malaria infection status monitoring [44, 45]. Infections with $P$. malariae [46], $P$. ovale [47] and $P$. vivax [48] are usually characterized by low parasitaemia levels, rendering these infections largely undetectable by using currently available RDT technologies [49].

In this study, more than three-quarter of $P$. falciparum and all non-falciparum infections were missed by the conventional diagnostic tools. A suitable screening method for malaria detection in blood units must have high sensitivity, high negative predictive value, must include all Plasmodium species, and should be cost efficient. Such a test must enable a reduction of TTM risk, as well as that of falsely deferred blood donors found to have false positive results.

The PlasQ malaria screening assay applied in this study meets some of these requirements. With a LOD of 0.05 parasites/ $\mu \mathrm{L}$, PlasQ has high sensitivity for $P$. falciparum and includes all other relevant Plasmodium species. This assay was extensively used during controlled human malaria infections in Tanzanian and Equatorial Guinean volunteers and no issues with specificity were observed (unpublished). As a limitation, qPCR analysis requires an advanced laboratory infrastructure, trained personnel and the costs for reagents and consumables for this assay is about USD 5, which is higher than the costs for RDT or microscopy-based malaria diagnosis.

This study was used to obtain more information about non-falciparum malaria species circulating in Malabo. Therefore a novel multiplex qPCR assay for parallel malaria parasite identification was developed. Using the PlasID assay, the main five human infectious malaria species can be detected qualitatively in a multiplex assay rendering it highly cost efficient since only one DNA extraction and qPCR reaction needs to be run. This novel malaria species identification assay should be used in combination with the Plas $Q$ assay, based on the finding that in the PlasID assay, a reduced sensitivity for $P$. falciparum is observed. Further development of diagnostic assays for the detection of $P$. malariae and $P$. ovale, should include the ability of species-specific quantification. Estimations based on Pspp18S target of the PlasQ assay, indicate that $P$. malariae and $P$. ovale single infections had a geometric mean density of about 0.6 parasites $/ \mu \mathrm{L}$, which is more than 14 times lower than $P$. falciparum single infections (8.8 parasites $/ \mu \mathrm{L})$.

Educating the general public in malaria endemic regions via TV, radio, and pamphlet about the existence of TTM and its potential health impact might help to raise the awareness of this malaria transmission route, both in potential blood donors and medical and nursing staff involved in blood transfusions [50]. This study confirms prior reports that replacement blood donors are particularly prone to harbour sub-patent malaria parasites [51]; hence this sub-population of donors are optimal for such awareness campaigns regarding potential TTM when donating blood for a family or friend. In addition to becoming aware of the potential TTM, medical and nursing staff should also use anti-malarial interventions when the potential for TTM is high and the malaria diagnostic infrastructure is limited. Ultimately, if the goal of malaria elimination is pursued vigorously, tackling TTM in sub-Saharan Africa needs to be included into the malaria elimination agenda.

\section{Conclusions}

More than one quarter of blood donations from healthy donors living in Malabo were infected with malaria parasites. Three species, including P. falciparum, $P$. malariae and $P$. ovale were detected using two different qPCR assays. The majority of these infections were not identified by the currently widely used malaria diagnostic tools such as RDTs and microscopy. Since the availability of molecular diagnostic methods in malaria endemic countries is still limited, the blood recipients living in malaria endemic countries should be treated following WHO recommendations.

\section{Additional files}

Additional file 1. Oligos used in this study.

Additional file 2. Identification of risk factors associated with a malaria positive blood donation. 


\section{Abbreviations}

RDT: rapid diagnostic test; HRP2: histidine rich protein 2; LDH: lactate dehydrogenase; LOD: limit of detection; NAT: nucleic acid amplification technology; qPCR: quantitative polymerase chain reaction; TTM: transfusion-transmitted malaria; EGMVI: Equatorial Guinea Malaria Vaccine Initiative; BIMCP: Bioko Island Malaria Control Project; Pspp18S: Pan-Plasmodium 18S rDNA sequence; PfvarATS: P. falciparum-specific acidic terminal sequence of the var genes.

\section{Authors' contributions}

Study concept and design: TS, TR, CM, CD. Acquisition of data: TS, TR, JS, LG, LA. Analyses and interpretation of data:TS, TR, MM, CD. Drafting the manuscript: TS, MM, CM, CD and all other authors reviewed the manuscript. Technical or material support: TR, JRB, GG, CC. Study supervision: MM, CM, CD. All authors read and approved the final manuscript.

\section{Author details}

${ }^{1}$ Department of Medical Parasitology and Infection Biology, Swiss Tropical and Public Health Institute, Basel, Switzerland. ${ }^{2}$ University of Basel, Basel, Switzerland. ${ }^{3}$ Malabo Blood Bank, Malabo, Equatorial Guinea. ${ }^{4}$ Equatorial Guinea Malaria Vaccine Initiative, Malabo, Equatorial Guinea. ${ }^{5}$ Bagamoyo Research and Training Centre, Ifakara Health Institute, Bagamoyo, United Republic of Tanzania. ${ }^{6}$ Medical Care Development International, Silver Spring, USA. ${ }^{7}$ Marathon EG Production Ltd, Malabo, Equatorial Guinea.

\section{Acknowledgements}

We would like to thank the voluntary and replacement blood donors at the Malabo blood bank for their willingness to donate blood and to save lives. We highly appreciate the support of Paul Lansdell, PHE Malaria Reference Laboratory and Don van Schalkwyk, Dept. of Immunology \& Infection, London School of Hygiene \& Tropical Medicine who kindly provided the DNA controls for non-falciparum malaria species. We would also like to thank Kenneth Mbwanji and Lukman Aroworamimo from Inqaba Biotec who kindly provided the GeneFinder Malaria kits, which were used to confirm the positive controls in this study.

\section{Competing interests}

The authors declare that they have no competing interests.

\section{Availability of data and materials}

The datasets supporting the conclusions of this article are included within the article.

\section{Consent for publication}

Not applicable.

\section{Ethics approval and consent to participate}

Approval for this study was given by the Ministry of Health and Social Welfare, Malabo, Bioko Norte, Republic of Equatorial Guinea. Study participants were assured of confidentiality and all data collected remained anonymous.

\section{Funding}

This study was supported by funds from the Equatorial Guinea Malaria Vaccine Initiative.

\section{Publisher's Note}

Springer Nature remains neutral with regard to jurisdictional claims in published maps and institutional affiliations.

Received: 6 September 2018 Accepted: 8 January 2019

Published online: 15 January 2019

\section{References}

1. WHO. World malaria report 2017. Geneva: World Health Organization; 2017

2. Ashley EA, Pyae Phyo A, Woodrow CJ. Malaria. Lancet. 2018;391:1608-21.

3. Nguyen T-N, von Seidlein L, Nguyen T-V, Truong P-N, Do Hung S, Pham $\mathrm{H}-\mathrm{T}$, et al. The persistence and oscillations of submicroscopic Plasmodium falciparum and Plasmodium vivax infections over time in Vietnam: an open cohort study. Lancet Infect Dis. 2018;18:565-72.

4. Bousema T, Okell L, Felger I, Drakeley C. Asymptomatic malaria infections: detectability, transmissibility and public health relevance. Nat Rev Microbiol. 2014;12:833-40.

5. Moody A. Rapid diagnostic tests for malaria parasites. Clin Microbiol Rev. 2002;15:66-78.

6. Boyce MR, O'Meara WP. Use of malaria RDTs in various health contexts across sub-Saharan Africa: a systematic review. BMC Public Health. 2017;17:470.

7. Mouatcho JC, Goldring JPD. Malaria rapid diagnostic tests: challenges and prospects. J Med Microbiol. 2013;62:1491-505.

8. Hendriksen ICE, Mtove G, Pedro AJ, Gomes E, Silamut K, Lee SJ, et al. Evaluation of a PfHRP2 and a pLDH-based rapid diagnostic test for the diagnosis of severe malaria in 2 populations of African children. Clin Infect Dis. 2011;52:1100-7.

9. Wongsrichanalai C, Barcus MJ, Muth S, Sutamihardja A, Wernsdorfer WH. A review of malaria diagnostic tools: microscopy and rapid diagnostic test (RDT). Am J Trop Med Hyg. 2007;77(Suppl. 6):119-27.

10. Srisutham S, Saralamba N, Malleret B, Rénia L, Dondorp AM, Imwong M. Four human Plasmodium species quantification using droplet digital PCR. PLOS ONE. 2017;12:e0175771.

11. Chew CH, Lim YAL, Lee PC, Mahmud R, Chua KH. Hexaplex PCR detection system for identification of five human Plasmodium species with an internal control. J Clin Microbiol. 2012:50:4012-9.

12. Hopkins H, Gonzalez IJ, Polley SD, Angutoko P, Ategeka J, Asiimwe C, et al Highly sensitive detection of malaria parasitemia in a malaria-endemic setting: performance of a new loop-mediated isothermal amplification kit in a remote clinic in Uganda. J Infect Dis. 2013;208:645-52.

13. Kamau E, Alemayehu S, Feghali KC, Saunders D, Ockenhouse CF. Multiplex $\mathrm{APCR}$ for detection and absolute quantification of malaria. PLOS ONE. 2013;8:e71539.

14. Kamau E, Tolbert LS, Kortepeter L, Pratt M, Nyakoe N, Muringo L, et al. Development of a highly sensitive genus-specific quantitative reverse transcriptase real-time PCR assay for detection and quantitation of Plasmodium by amplifying RNA and DNA of the $18 \mathrm{~S}$ rRNA genes. J Clin Microbiol. 2011;49:2946-53.

15. Murphy SC, Prentice JL, Williamson K, Wallis CK, Fang FC, Fried M, et al. Real-time quantitative reverse transcription PCR for monitoring of bloodstage Plasmodium falciparum infections in malaria human challenge trials. Am J Trop Med Hyg. 2012;86:383-94.

16. Adams M, Joshi SN, Mbambo G, Mu AZ, Roemmich SM, Shrestha B, et al. An ultrasensitive reverse transcription polymerase chain reaction assay to detect asymptomatic low-density Plasmodium falciparum and Plasmodium vivax infections in small volume blood samples. Malar J. 2015:14:520.

17. Hofmann N, Mwingira F, Shekalaghe S, Robinson LJ, Mueller I, Felger I. Ultra-sensitive detection of Plasmodium falciparum by amplification of multi-copy subtelomeric targets. PLoS Med. 2015;12:e1001788.

18. Mangold KA, Manson RU, Koay ESC, Stephens L, Regner M, Thomson RBJ, et al. Real-time PCR for detection and identification of Plasmodium spp. J Clin Microbiol. 2005;43:2435-40.

19. WHO. Screening donated blood for transfusion-transmissible infections: recommendation. Geneva: World Health Organization; 2010.

20. WHO. Blood donor selection: guidelines on assessing donor suitability for blood donation. Geneva: World Health Organization; 2012.

21. Freimanis G, Sedegah M, Owusu-Ofori S, Kumar S, Allain J-P. Investigating the prevalence of transfusion transmission of Plasmodium within a hyperendemic blood donation system. Transfusion. 2013;53:1429-41.

22. Owusu-Ofori AK, Betson M, Parry CM, Stothard JR, Bates I. Transfusion transmitted malaria in Ghana. Clin Infect Dis. 2013;56:1735-41.

23. Lacerda MVG, Monteiro WM, Alexandre MAA, Alho RRM, Kiesslich D, Fraiji NA. We need to talk more about transfusion-transmitted malaria in Plasmodium vivax endemic areas. Rev Bras Hematol Hemoter. 2014:36:385-7.

24. Scuracchio P, Vieira SD, Dourado DA, Bueno LM, Colella R, Ramos-Sanchez EM, et al. Transfusion-transmitted malaria: case report of asymptomatic donor harboring Plasmodium malariae. Rev Instit Med Trop de São Paulo. 2011:53:55-9.

25. Bird EM, Parameswaran U, William T, Khoo TM, Grigg MJ, Aziz A, et al. Transfusion-transmitted severe Plasmodium knowlesi malaria in a 
splenectomized patient with beta-thalassaemia major in Sabah, Malaysia: a case report. Malar J. 2016;15:357.

26. Martin-Davila P, Norman F, Fortun-Abete J, Piris M, Lovatti R, Rubio JM, et al. Donor-derived multiorgan transmission of mixed P. malariae and P. ovale infection: impact of globalization on post-transplant infections. Transpl Infect Dis. 2018;20:e12938.

27. Guerra-Neira A, Rubio JM, Royo JR, Ortega JC, Auñón AS, Diaz PB, et al. Plasmodium diversity in non-malaria individuals from the Bioko Island in Equatorial Guinea (West Central-Africa). Int J Health Geogr. 2006:5:27.

28. Mendes C. Duffy negative antigen is no longer a barrier to Plasmodium vivax - molecular evidences from the African West Coast (Angola and Equatorial Guinea). PLoS Negl Trop Dis. 2011;5:e1192.

29. Berzosa PJ, García ML, Micó M, Edú M, Rubio JM, Alvar J, et al. Seminested, multiplex polymerase chain reaction for detection of human malaria parasites and evidence of Plasmodium vivax infection in Equatorial Guinea. Am J Trop Med Hyg. 1999;60:183-7.

30. Cook J, Hergott D, Phiri W, Rivas MR, Bradley J, Segura L, et al. Trends in parasite prevalence following 13 years of malaria interventions on Bioko island, Equatorial Guinea: 2004-2016. Malar J. 2018;17:62.

31. Olotu A, Urbano V, Hamad A, Eka M, Chemba M, Nyakarungu E, et al. Advancing global health through development and clinical trials partnerships: a randomized, placebo-controlled, double-blind assessment of safety, tolerability, and immunogenicity of PfSPZ vaccine for malaria in healthy Equatoguinean men. Am J Trop Med Hyg. 2018;98:308-18.

32. Kamau E, Alemayehu S, Feghali KC, Komisar J, Regules J, Cowden J, et al. Measurement of parasitological data by quantitative real-time PCR from controlled human malaria infection trials at the Walter Reed Army Institute of Research. Malar J. 2014;13:288.

33. Cnops L, Jacobs J, Van Esbroeck M. Validation of a four-primer real-time PCR as a diagnostic tool for single and mixed Plasmodium infections. Clin Microbiol Infect. 2011:17:1101-7.

34. Phuong M, Lau R, Ralevski F, Boggild AK. Sequence-based optimization of a quantitative real-time PCR assay for detection of Plasmodium ovale and Plasmodium malariae. J Clin Microbiol. 2014;52:1068-73.

35. Reller ME, Chen WH, Dalton J, Lichay MA, Dumler JS. Multiplex 5 ' nuclease quantitative real-time PCR for clinical diagnosis of malaria and specieslevel identification and epidemiologic evaluation of malaria-causing parasites, including Plasmodium knowlesi. J Clin Microbiol. 2013;51:2931-8.

36. Miller RH, Obuya CO, Wanja EW, Ogutu B, Waitumbi J, Luckhart S, et al. Characterization of Plasmodium ovale curtisi and P. ovale wallikeri in Western Kenya utilizing a novel species-specific real-time PCR assay. PLoS Negl Trop Dis. 2015;9:e0003469.

37. Divis PCS, Shokoples SE, Singh B, Yanow SK. A TaqMan real-time PCR assay for the detection and quantitation of Plasmodium knowlesi. Malar J. 2010;9:344
38. Allain JP, Owusu-Ofori AK, Assennato SM, Marschner S, Goodrich RP, Owusu-Ofori S. Effect of Plasmodium inactivation in whole blood on the incidence of blood transfusion-transmitted malaria in endemic regions: the African Investigation of the Mirasol System (AIMS) randomised controlled trial. Lancet. 2016;387:1753-61.

39. Guindo A, Touré BA, Guindo P, Baraika MA, Sarro YS, Fané B, et al. Transmission of Plasmodium falciparum by red blood cell transfusions in the management of sickle cell disease patients in Mali. Transfus Med. 2016:26:153-5.

40. Allain J-P, Assennato SM, Osei EN, Owusu-Ofori AK, Marschner S, Goodrich RP, et al. Characterization of posttransfusion Plasmodium falciparum infection in semi-immune nonparasitemic patients. Transfusion. 2016:56:2374-83.

41. Verra F, Angheben A, Martello E, Giorli G, Perandin F, Bisoffi Z. A systematic review of transfusion-transmitted malaria in non-endemic areas. Malar J. 2018;17:36

42. Chattopadhyay R, Majam VF, Kumar S. Survival of Plasmodium falciparum in human blood during refrigeration. Transfusion. 2011:51:630-5.

43. Das S, Jang IK, Barney B, Peck R, Rek JC, Arinaitwe E, et al. Performance of a high-sensitivity rapid diagnostic test for Plasmodium falciparum malaria in asymptomatic individuals from Uganda and Myanmar and naive human challenge infections. Am J Trop Med Hyg. 2017;97:1540-50.

44. Maltha J, Gillet P, Jacobs J. Malaria rapid diagnostic tests in endemic settings. Clin Microbiol Infect. 2013;19:399-407.

45. Lover AA, Baird JK, Gosling R, Price R. Malaria elimination: time to target all species. Am J Trop Med Hyg. 2018;99:17-23.

46. Collins WE, Jeffery GM. Plasmodium malariae: parasite and disease. Clin Microbiol Rev. 2007;20:579-92.

47. Collins WE, Jeffery GM. Plasmodium ovale: parasite and disease. Clin Microbiol Rev. 2005;18:570-81.

48. Howes RE, Battle KE, Mendis KN, Smith DL, Cibulskis RE, Baird JK, et al. Global epidemiology of Plasmodium vivax. Am J Trop Med Hyg. 2016;95(6 Suppl):15-34

49. Maltha J, Gillet P, Jacobs J. Malaria rapid diagnostic tests in travel medicine. Clin Microbiol Infect. 2013;19:408-15.

50. Owusu-Ofori A, Gadzo D, Bates I. Transfusion-transmitted malaria: donor prevalence of parasitaemia and a survey of healthcare workers knowledge and practices in a district hospital in Ghana. Malar J. 2016;15:234.

51. Lund TC, Hume H, Allain JP, McCullough J, Dzik W. The blood supply in sub-Saharan Africa: needs, challenges, and solutions. Transfus Apher Sci. 2013;49:416-21.

\footnotetext{
Ready to submit your research? Choose BMC and benefit from:

- fast, convenient online submission

- thorough peer review by experienced researchers in your field

- rapid publication on acceptance

- support for research data, including large and complex data types

- gold Open Access which fosters wider collaboration and increased citations

- maximum visibility for your research: over $100 \mathrm{M}$ website views per year
}

At BMC, research is always in progress.

Learn more biomedcentral.com/submissions 